\title{
On the family of Thue equations
}

$$
x^{3}-(n-1) x^{2} y-(n+2) x y^{2}-y^{3}=k
$$

by

\section{Mignotte (Strasbourg), A. Pethő (Debrecen) and F. LEMMERMEYER (Heidelberg)}

1. Introduction. The family of cubic Thue equations $x^{3}-(n-1) x^{2} y-$ $(n+2) x y^{2}-y^{3}= \pm 1$, with $n \geq 0$, was studied by E. Thomas. He proved in [T1] that it has only "trivial solutions" except for a finite number of values of the parameter $n$, explicitly for $n<10^{8}$. Then M. Mignotte $[\mathrm{M}]$ solved completely this family of equations and proved that non-trivial solutions occur only for $n \leq 3$.

Next, other infinite families of Thue equations $F(x, y)= \pm 1$ were studied. In the cubic case there are works of Thomas [T2], Mignotte and Tzanakis [MT]; in these cases the family was completely solved except for an explicit finite range for the parameter. In the quartic case, there are works of Pethő [P2], Lettl and Pethő [LeP] and Mignotte, Pethő and Roth [MPR], where the last two studies were completely finished.

Here, we come back to the family of cubics investigated by E. Thomas, the case where $F_{n}(x, y)=x^{3}-(n-1) x^{2} y-(n+2) x y^{2}-y^{3}$, but we add a new parameter: the number $k$ on the right hand side. In other words, we study a family of cubic diophantine equations which depend on two parameters.

Our results are explicit bounds for the integer solutions in terms of the two parameters $n$ and $k$; and we give a special attention to the case $x^{3}-$ $(n-1) x^{2} y-(n+2) x y^{2}-y^{3}= \pm(2 n+1)$. This case has some interest since we prove that the diophantine equation $F_{n}(x, y)=k$ has no solution in the range $1<|k|<2 n+1$ unless $k$ is a cube (the existence of a solution in this case is trivial).

We formulate now the three theorems which are the main results of the present paper.

Research of the second author supported in part by Hungarian National Foundation for Scientific Research Grant No. 16791/95. 
Theorem 1. Let $n \geq 1650, k$ be integers. If

$$
\left|x^{3}-(n-1) x^{2} y-(n+2) x y^{2}-y^{3}\right|=k
$$

for some $x, y \in \mathbb{Z}$, then

$$
\log |y|<c_{1} \log ^{2}(n+2)+c_{2} \log n \log k,
$$

where

$$
c_{1}=700+476.4\left(1-\frac{1432.1}{n}\right)^{-1}\left(1.501-\frac{1902}{n}\right)<1956.4
$$

and

$$
c_{2}=29.82+\left(1-\frac{1432.1}{n}\right)^{-1} \frac{1432}{n \log n}<30.71 .
$$

It is well known (see $[\mathrm{GyP}]$ and the references therein) that the general estimates of the solutions of a Thue equation depend polynomially on the constant term, but exponentially on the height of the form itself, i.e. the maximum of the absolute values of the coefficients of the form corresponding to the Thue equation. In the present case, the dependence is polynomial in $k$ and "nearly" polynomial in the height of $F_{n}(x, y)$ (which is $\left.n+2\right)$. Moreover, the constants appearing here are much better than in other similar results (see e.g. [BSt]).

To illustrate how sharp is the estimate of Theorem 1 take $n=1650$ and $k=10^{9}$. Then we get

$$
|y|<10^{48698} .
$$

This is certainly a bound which is reachable with the present numerical techniques, and in any case such a bound is much smaller than previous bounds obtained for cubic Thue equations.

From Theorem 1, we are able to derive an effective improvement of Liouville's inequality for the rational approximations of the zeros of $F_{n}(x, 1)$.

TheOREM 2. Let $\lambda$ be one of the zeros of $F_{n}(x, 1)$. If $n \geq 1650$ and $\lambda \in]-1,0[$ or $\lambda \in]-2,-1[$ then

$$
\left|\lambda-\frac{x}{y}\right|>(n+2)^{-c_{3}} y^{-3+1 /\left(c_{2} \log n\right)}
$$

and if $\lambda \in(n, n+1)$ then

$$
\left|\lambda-\frac{x}{y}\right|>(n+2)^{-c_{3}-1} y^{-3+1 /\left(c_{2} \log n\right)}
$$

for all $(x, y) \in \mathbb{Z}^{2}, y \neq 0$, where

$$
c_{3}=c_{1} \frac{\log (n+2)}{\log n}+1<64.72 .
$$


In the second part of the paper we deal with the inequality

$$
\left|x^{3}-(n-1) x^{2} y-(n+2) x y^{2}-y^{3}\right| \leq 2 n+1 .
$$

Combining the method of the proof of Theorem 1 with a result of Lemmermeyer and Pethö $[\mathrm{LP}]$ we are able to solve the above inequality completely and prove the following.

THEOREM 3. Let $n$ be a non-negative integer. If $(x, y) \in \mathbb{Z}^{2}$ is a solution of equation (1), then either $(x, y)=t(u, v)$ with an integer $0 \leq|t| \leq \sqrt[3]{2 n+1}$ and $\pm(u, v) \in\{(1,0),(0,1),(-1,1)\}$ or $\pm(x, y) \in\{(-1,-1),(-1,2)$, $(-1, n+1),(-n,-1),(n+1,-n),(2,-1)\}$, except when $n=2$, in which case (1) has the extra solutions: $\pm(x, y) \in\{(-4,3),(8,3),(1,-4),(3,1),(3,-11)\}$.

The plan of this paper is the following. Section 2 contains two general lemmas. The next section is devoted to a pure numerical study of the roots of the polynomials $x^{3}-(n-1) x^{2}-(n+2) x-1$; these estimates are used in the linear forms of logs which are studied in the sequel. Section 4, which is short and easy, contains the proof of Theorem 2 assuming Theorem 1.

The next section is an instance of the classical Siegel-Baker reduction of a Thue equation to a linear form in logs. This study is detailed in order to lead to sharp estimates; here we get linear forms in three logs. Then the linear form in logs obtained in Section 5 is studied a first time in Section 6. In this study, this linear form is considered as a linear form in two logs (we just group two terms). To get sharp estimates, we apply the general result of Laurent-Mignotte-Nesterenko. Of course, this implies a tedious reconstruction of the proof of the lower bound of this linear form; for example we have to choose the parameters of the "auxiliary function" (more exactly, of the interpolation determinant): this is the price to pay. The conclusion of this section is an upper bound on $y$, in terms of the heights of the terms of the linear form. The very long Section 6 contains a second study of this linear form in "two" logs; first, we prove an estimate of the coefficients of this linear form in terms of $y$, then we bound these coefficients and (after some computation) at the end we get an explicit upper bound of $y$, in terms of the initial parameters $n$ and $k$. This ends the proof of Theorem 1 .

Section 8, which is short and easy, gives a simple upper bound of $y$ depending only on $n$, when $k<n^{4}$; this result is used in the next section.

The last two sections deal with the proof of Theorem 3. Here, a result of Lemmermeyer and Pethő [LP] plays an essential role. Section 9 contains a study of the special linear form in "two" logs which occurs in this case; this leads to the proof of Theorem 3 for $n>1700$. To cover the range $n \leq 1700$, in Section 10 we consider - for the first time - the linear form in logs as a linear form in three logs. This study is classical and we can easily finish the proof of Theorem 3. 
2. Preliminary lemmas. This section contains two lemmas on cubic fields and cubic forms which have a general interest.

Lemma 1 [P1, Theorem 2]. Let $f(X, Y)=X^{3}+b X^{2} Y+c X Y^{2}+d Y^{3}$ be a cubic form with positive discriminant $D_{f}$, and suppose that $f(x, y)=k$, where $x, y$ are rational integers, $y \neq 0$. Let $\alpha_{i}, i=1,2,3$, be the roots of the polynomial $f(X, 1)$. Put $L_{i}=\alpha_{i}-x / y, i=1,2,3$, and suppose that $\left|L_{1}\right| \leq\left|L_{2}\right| \leq\left|L_{3}\right|$. Then

$$
\left|L_{1}\right|^{4}\left|L_{2}\right|^{2} \leq \frac{4|k|^{4}}{D_{f}|y|^{12}}
$$

Pr o of. Notice the formula

$$
D_{f}=\prod_{i<j}\left(\alpha_{i}-\alpha_{j}\right)^{2}=L_{3}^{4} L_{2}^{2}\left(1-\frac{L_{2}}{L_{3}}\right)^{2}\left(1-\frac{L_{1}}{L_{3}}\right)^{2}\left(1-\frac{L_{1}}{L_{3}}\right)^{2} .
$$

Since the function $g(u, v)=(1-u)(1-v)(1-u / v)$ satisfies $0 \leq g(u, v) \leq 2$ in the domain $-1 \leq u, v \leq 1,|v| \leq|u|$, we have $D_{f} \leq 4 L_{3}^{4} L_{2}^{2}$. Multiplying both sides of this inequality by $L_{2}^{2} L_{1}^{4}$ and using the relation $L_{1} L_{2} L_{3}=k / y^{3}$, we get the result.

Corollary 1. Using $\left|L_{1}\right| \leq\left|L_{2}\right|$, we get

$$
\left|L_{1}\right| \leq\left(\frac{4 k^{4}}{D_{f}}\right)^{1 / 6} \frac{1}{|y|^{2}} .
$$

Lemma 2. Let $\alpha \in K$, where $K$ is a cubic Galois field. Denote by $\alpha, \alpha^{\prime}$ and $\alpha^{\prime \prime}$ the conjugates of $\alpha$, and relabel them $\alpha_{1}, \alpha_{2}, \alpha_{3}$ in such a way that $\left|\alpha_{1}\right| \geq\left|\alpha_{2}\right| \geq\left|\alpha_{3}\right|$. Then the measure of the quotient $\alpha / \alpha^{\prime}$ satisfies

$$
M\left(\alpha / \alpha^{\prime}\right) \leq\left|\alpha_{1}\right|^{2}\left|\alpha_{2}\right| .
$$

Proof. Let $k$ be the norm of $\alpha$. Consider the polynomial

$$
k\left(X-\alpha / \alpha^{\prime}\right)\left(X-\alpha^{\prime} / \alpha^{\prime \prime}\right)\left(X-\alpha^{\prime \prime} / \alpha\right)=\left(\alpha^{\prime} X-\alpha\right)\left(\alpha^{\prime \prime} X-\alpha^{\prime}\right)\left(\alpha X-\alpha^{\prime \prime}\right) .
$$

Clearly, this polynomial has rational integer coefficients, and $\alpha / \alpha^{\prime}$ is a root of it. Thus,

$$
\begin{aligned}
M\left(\alpha / \alpha^{\prime}\right) & \leq|k| \max \left\{1,\left|\alpha / \alpha^{\prime}\right|\right\} \max \left\{1,\left|\alpha^{\prime} / \alpha^{\prime \prime}\right|\right\} \max \left\{1,\left|\alpha^{\prime \prime} / \alpha\right|\right\} \\
& =k\left|\alpha_{1}\right| /\left|\alpha_{3}\right|=\left|\alpha_{1}\right|^{2}\left|\alpha_{2}\right| .
\end{aligned}
$$

R e m ark. It is easy to verify that the previous lemma can be generalized in the following way: Let $\alpha \in K$, where $K$ is a cyclic Galois field of degree $d$. Let $\alpha^{\prime}$ be some conjugate of $\alpha$, with $\alpha^{\prime} \neq \alpha$. Choose an order on the set $\alpha_{1}, \ldots, \alpha_{d}$ of conjugates of $\alpha$ for which $\left|\alpha_{1}\right| \geq\left|\alpha_{2}\right| \geq \ldots \geq\left|\alpha_{d}\right|$. Then the measure of the quotient $\alpha / \alpha^{\prime}$ satisfies

$$
M\left(\alpha / \alpha^{\prime}\right) \leq\left|\alpha_{1}\right|^{d-1}\left|\alpha_{2}\right|^{d-2} \ldots\left|\alpha_{d-1}\right| .
$$


3. Numerical study of the roots of $x^{3}-(n-1) x^{2}-(n+2) x-1$. In this section, we gather several sharp estimates for the roots of the polynomial $f$ associated with the cubic form

$$
F(x, y)=F_{n}(x, y)=x^{3}-(n-1) x^{2} y-(n+2) x y^{2}-y^{3},
$$

that is,

$$
f(x)=f_{n}(x)=x^{3}-(n-1) x^{2}-(n+2) x-1 .
$$

From now on, we shall keep these definitions for $f, f_{n}, F_{n}$ and $F$.

Since $f(-2)<0, f(-1)=1, f(0)=-1, f(n)=-2 n-1$ and $f(n+1)=$ $n^{2}+n+1$, this polynomial has three real roots, say $\lambda=\lambda^{(1)}, \lambda^{(2)}, \lambda^{(3)}$, and we may suppose that

$$
\lambda \in]-1,0\left[, \quad \lambda^{(2)} \in\right]-2,-1\left[, \quad \lambda^{(3)} \in\right] n, n+1[.
$$

Moreover, the polynomial $f$ is invariant under the change of variable $\sigma$ : $x \mapsto-1 /(1+x)$, which satisfies $\sigma^{2}(x)=-(1+x) / x$ and $\sigma^{3}(x)=x$. This proves that the field $\mathbb{Q}(\lambda)$ is a Galois field and that

$$
\lambda^{(2)}=-\frac{1}{\lambda+1} \quad \text { and } \quad \lambda^{(3)}=-\left(1+\frac{1}{\lambda}\right) .
$$

More generally, $\sigma^{j}\left(\lambda^{(i)}\right)=\lambda^{(k)}$, where $k \equiv i+j \bmod 3$.

Since

$$
f_{n}\left(n+\frac{2}{n}\right)=1+\frac{4}{n}+\frac{4}{n^{2}}+\frac{8}{n^{3}},
$$

we see that

$$
\lambda^{(3)}<n+\frac{2}{n} .
$$

A second computation gives

$$
f_{n}\left(n+\frac{2}{n+1}\right)=-1+\frac{4}{n+1}-\frac{4}{(n+1)^{2}}+\frac{8}{(n+1)^{3}},
$$

which shows that

$$
\lambda^{(3)}>n+\frac{2}{n+1}
$$

for $n>2$. In the sequel, we suppose $n \geq 3$, and the two cases $n=1$ and $n=2$ will be considered separately.

In terms of $\lambda^{(3)}$, we have

$$
\lambda^{(3)}=-\left(1+\frac{1}{\lambda}\right) \Rightarrow \lambda=-\frac{1}{\lambda^{(3)}+1}
$$

and

$$
\lambda^{(2)}=-\frac{1}{1+\lambda}=-\frac{1}{1-\frac{1}{\lambda^{(3)}+1}}=-\frac{\lambda^{(3)}+1}{\lambda^{(3)}} .
$$


These estimates imply (for $n \geq 3$ )

$$
-\frac{n+1}{n^{2}+2 n+3}=-\frac{1}{1+n+\frac{2}{n+1}}<\lambda<-\frac{1}{1+n+\frac{2}{n}}=-\frac{n}{n^{2}+n+2}
$$

and

$$
-1-\frac{n+1}{n^{2}+n+2}<\lambda^{(2)}=-\left(1+\frac{1}{\lambda^{(3)}}\right)<-1-\frac{n}{n^{2}+2} .
$$

These estimates lead to estimates for logarithms. Indeed,

$$
\begin{aligned}
& \log n+\frac{2}{n(n+1)}-\frac{2}{(n(n+1))^{2}}<\log \left(n+\frac{2}{n+1}\right)<l_{3}:=\log \lambda^{(3)} \\
&<\log \left(n+\frac{2}{n}\right)<\log n+\frac{2}{n^{2}} \\
& \frac{n-1 / 2}{n^{2}+2}<\frac{n}{n^{2}+2}-\frac{n^{2}}{2\left(n^{2}+2\right)^{2}}<l_{2}:=\log \left|\lambda^{(2)}\right|<\frac{n+1}{n^{2}+n+2},
\end{aligned}
$$

and

$-\log (n+1)-\frac{2}{n(n+1)}<l_{1}:=\log |\lambda|<-\log (n+1)-\frac{2}{(n+1)^{2}}+\frac{2}{(n+1)^{4}}$.

4. Proof of Theorem 2. Assuming that Theorem 1 is true we now give the proof of Theorem 2 because it is short and depends only on the estimates of Section 3. We consider only the case $\lambda \in] 1,0[$. The proofs of the other cases are similar. Assume that there exists $(x, y) \in \mathbb{Z}^{2}, y \neq 0$, such that

$$
|\lambda-x / y| \leq(n+2)^{-c_{3}} y^{-3+1 /\left(c_{2} \log n\right)} .
$$

Let $\lambda_{2}$ and $\lambda_{3}$ denote the other zeros of $F_{n}(x, 1)$ such that $\left.\lambda_{2} \in\right]-2,-1[$ and $\left.\lambda_{3} \in\right] n, n+1[$. Then

$$
\left|\lambda_{i}-x / y\right| \leq\left|\lambda-\lambda_{i}\right|+(n+2)^{-c_{3}}
$$

for $i=2,3$. Using these inequalities and the estimates of Section 3, one can easily prove that

$$
\left|\lambda_{2} y-x\right| \cdot\left|\lambda_{3} y-x\right|<(n+2)|y|
$$

hence

$$
\left|F_{n}(x, y)\right|<(n+2)^{-\left(c_{3}-1\right)}|y|^{1 /\left(c_{2} \log n\right)} .
$$

Putting $k=(n+2)^{-\left(c_{3}-1\right)}|y|^{1 /\left(c_{2} \log n\right)}$, Theorem 1 implies

$$
\begin{aligned}
\log |y| & <c_{1} \log ^{2}(n+2)+c_{2} \log n \log k \\
& <c_{1} \log ^{2}(n+2)+c_{2} \log n\left(-c_{1} \frac{\log ^{2}(n+2)}{c_{2} \log n}+\frac{\log |y|}{c_{2} \log n}\right)=\log |y|,
\end{aligned}
$$

a contradiction, and the theorem is proved. 
5. Reduction of the Thue equation $F_{n}(x, y)=k$. Let $x, y$ be rational integers, $y>0$, such that

$$
F_{n}(x, y)=x^{3}-(n-1) x^{2} y-(n+2) x y^{2}-y^{3}= \pm k, \quad k>0 .
$$

The condition $k>0$ is not restrictive since $F(-x,-y)=-F(x, y)$.

We know (see [T1]) that $\lambda, \lambda^{(2)}$ constitute a fundamental system of units for the order $\mathbb{Z}[\lambda]$.

The following lemma is useful for the reduction of the Thue equation in the case $k>1$.

Lemma 3. Let $0 \neq \beta \in \mathbb{Z}[\lambda]$ and let $0<c_{1}, c_{2} \in \mathbb{R}$. Then there exist rational integers $a_{1}$ and $a_{2}$ and some element $\gamma \in \mathbb{Z}[\lambda]$ such that

$$
\beta=\gamma \lambda^{a_{1}}\left(\lambda^{(2)}\right)^{a_{2}}
$$

with, for $n \geq 3$,

$$
c_{i} \leq\left|\gamma^{(i)}\right|<(n+3) c_{i}, \quad i=1,2, \quad \text { and } \quad \frac{|N(\beta)|}{(n+3)^{2} c_{1} c_{2}}<\left|\gamma^{(3)}\right| \leq \frac{|N(\beta)|}{c_{1} c_{2}}
$$

Proof. Put

$$
\widetilde{c}_{i}=c_{i} \exp \left\{\frac{1}{2}|\log | \lambda^{(i)}||+\frac{1}{2}|\log | \lambda^{(i+1)}||\right\}, \quad i=1,2,
$$

and consider the system of equations

$$
\log \left|\beta^{(i)} / \widetilde{c}_{i}\right|=r_{1} \log \left|\lambda^{(i)}\right|+r_{2} \log \left|\lambda^{(i+1)}\right|, \quad i=1,2 .
$$

As $\left(\log \left|\lambda^{(1)}\right|, \log \left|\lambda^{(2)}\right|, \log \left|\lambda^{(3)}\right|\right)$ and $\left(\log \left|\lambda^{(2)}\right|, \log \left|\lambda^{(3)}\right|, \log \left|\lambda^{(1)}\right|\right)$ are linearly independent vectors in $\mathbb{R}^{3}$, this system has a unique real solution, $r_{1}$ and $r_{2}$. Put $r_{1}=a_{1}+\theta_{1}$ and $r_{2}=a_{2}+\theta_{2}$, where $a_{1}, a_{2} \in \mathbb{Z}$, and $\left|\theta_{1}\right|,\left|\theta_{2}\right| \leq 1 / 2$. Then consider the algebraic number $\gamma=\beta \lambda^{-a_{1}}\left(\lambda^{(2)}\right)^{-a_{2}}$. Then $|N \gamma|=|N \beta|$ and $\gamma \in \mathbb{Z}[\lambda]$, and we get

$$
|\log | \gamma^{(i)} / \widetilde{c}_{i}|| \leq \frac{1}{2}\left(|\log | \lambda^{(i)}||+|\log | \lambda^{(i+1)}||\right), \quad i=1,2,
$$

which implies

$$
\log c_{i}<\log \left|\gamma^{(i)}\right| \leq \log c_{i}+\left(|\log | \lambda^{(i)}||+|\log | \lambda^{(i+1)}||\right), \quad i=1,2 .
$$

By the estimates of the previous section, for $n \geq 3$,

$$
\begin{aligned}
|\log | \lambda^{(1)}||+|\log | \lambda^{(2)}|| & =\log \left(\lambda^{(3)}+1\right)+\log \left(1+\frac{1}{\lambda^{(3)}}\right) \\
& =\log \left(\lambda^{(3)}+2+\frac{1}{\lambda^{(3)}}\right) \\
& <\log \left(n+\frac{2}{n}+2+\frac{n}{n^{2}+2}\right)<\log (n+3),
\end{aligned}
$$


and

$$
\begin{aligned}
|\log | \lambda^{(2)}||+|\log | \lambda^{(3)}|| & =\log \left(\lambda^{(3)}\right)+\log \left(1+\frac{1}{\lambda^{(3)}}\right) \\
& =\log \left(\lambda^{(3)}+1\right)<\log (n+2) .
\end{aligned}
$$

This proves the first two inequalities. The third follows immediately.

Corollary 2. Let $0 \neq \beta \in \mathbb{Z}[\lambda]$ with norm $k$. Then there exist rational integers $a_{1}$ and $a_{2}$ and some element $\gamma \in \mathbb{Z}[\lambda]$ such that

$$
\beta=\gamma \lambda^{a_{1}}\left(\lambda^{(2)}\right)^{a_{2}}
$$

with

$$
M\left(\gamma^{(2)} / \gamma^{(3)}\right) \leq k(n+3)^{5 / 2} \quad \text { and } \quad|\log | \gamma^{(2)} / \gamma^{(3)}|| \leq \frac{3}{2} \log (n+3) .
$$

Proof. Put $c_{1}=c_{2}=\sqrt[3]{k} / \sqrt{n+3}$. The application of Lemma 3 implies the existence of $\gamma \in \mathbb{Z}[\lambda]$, which is associated with $\beta$ and which satisfies

$$
\sqrt[3]{k} /(n+3)<\left|\gamma^{(3)}\right|<\sqrt[3]{k}(n+3) .
$$

From Lemma 2 , we get $M\left(\gamma^{(2)} / \gamma^{(3)}\right) \leq k(n+3)^{5 / 2}$. Finally, as $\log \left|\gamma^{(2)}\right|-\log \left|\gamma^{(3)}\right| \leq \frac{1}{3} \log k+\log (n+3)-\frac{1}{3} \log k+\frac{1}{2} \log (n+3)=\frac{3}{2} \log (n+3)$ and

$$
\begin{aligned}
\log \left|\gamma^{(2)}\right|-\log \left|\gamma^{(3)}\right| & \geq \frac{1}{3} \log k-\log (n+3)-\frac{1}{3} \log k-\frac{1}{2} \log (n+3) \\
& =-\frac{3}{2} \log (n+3),
\end{aligned}
$$

the statement is proved.

If $(x, y)$ is a solution of $(2)$, then there exist $u_{1}, u_{2} \in \mathbb{Z}$ such that

$$
x-\lambda y=\gamma \lambda^{u_{1}}\left(\lambda^{(2)}\right)^{u_{2}},
$$

where $\gamma$ satisfies the conditions of the preceding corollary.

We may assume (see Thomas [T1]) that $|x / y-\lambda| \leq\left|x / y-\lambda^{(2)}\right|$, $\left|x / y-\lambda^{(3)}\right|$. Applying Lemma 1 a first time, we get

$$
\left|L_{1}\right| \leq\left(\frac{4 k^{4}}{\left(n^{2}+n+7\right)^{2}}\right)^{1 / 6} \frac{1}{y^{2}},
$$

using the fact that the discriminant of the form $F_{n}$ is equal to $\left(n^{2}+n+7\right)^{2}$.

We suppose that $n>0$ and $y \geq \max \{3, k, n\}$. Then the previous inequality implies $\left|L_{1}\right| \leq 1 / 7$, thus

$$
\left|L_{2}\right| \geq\left|\lambda^{(2)}-\lambda\right|-\left|L_{1}\right|>3 / 4,
$$

and a second application of Lemma 1 gives

$$
|x-\lambda y| \leq\left(\frac{64 k^{4}}{9\left(n^{2}+n+7\right)^{2}}\right)^{1 / 4} \frac{1}{|y|^{2}} \leq \frac{0.55}{y} \leq 0.19 .
$$


Notice also that, for $n \geq 3$,

$$
\begin{gathered}
y \leq\left|x-\lambda^{(2)} y\right| \leq y\left(1+\frac{2}{n^{2}}\right), \\
y\left(n+\frac{2}{n}\right) \leq\left|x-\lambda^{(3)} y\right| \leq y\left(n+\frac{4}{n}\right) .
\end{gathered}
$$

Let us give the proofs of these two pairs of inequalities. By the estimates of the preceding section, we have

$$
\lambda^{(2)}-\lambda=-\frac{\lambda^{(3)}+1}{\lambda^{(3)}}+\frac{1}{\lambda^{(3)}+1}=-1-\frac{1}{\lambda^{(3)}\left(\lambda^{(3)}+1\right)},
$$

hence

$$
1+\frac{1}{(n+2)^{2}}<\left|\lambda^{(2)}-\lambda\right|<1+\frac{1}{n^{2}} .
$$

This leads to the estimate

$$
\begin{aligned}
y & \leq\left(1+\frac{1}{(n+2)^{2}}\right) y-\frac{0.55}{y} \leq\left|\lambda-\lambda^{(2)}\right| y-|x-\lambda y| \leq\left|x-\lambda^{(2)} y\right| \\
& \leq\left|\lambda-\lambda^{(2)}\right| y+|x-\lambda y| \leq \frac{0.55}{y}+\left(1+\frac{1}{n^{2}}\right) y \leq y\left(1+\frac{2}{n^{2}}\right) .
\end{aligned}
$$

In the same way,

$$
\begin{aligned}
n+\frac{3}{n+1} & <n+\frac{2}{n+1}+\frac{n+1}{n^{2}+n+2}<\lambda^{(3)}-\lambda=\lambda^{(3)}+\frac{1}{\lambda^{(3)}+1} \\
& <n+\frac{2}{n}+\frac{n}{n^{2}+2}<n+\frac{3}{n},
\end{aligned}
$$

which implies

$$
\left(n+\frac{2}{n}\right) y<\left|x-\lambda^{(3)} y\right|<\left(n+\frac{4}{n}\right) y .
$$

Now, using (5), we can refine (4),

$$
|x-\lambda y|=\frac{k}{\left|x-\lambda^{(2)} y\right| \cdot\left|x-\lambda^{(3)} y\right|}<\frac{k}{n y^{2}} .
$$

Considering the conjugates of (3), we get Siegel's identity

$$
\begin{aligned}
\left(\lambda^{(3)}-\lambda\right) \gamma^{(2)}\left(\lambda^{(2)}\right)^{u_{1}}\left(\lambda^{(3)}\right)^{u_{2}}-\left(\lambda^{(2)}-\lambda\right) \gamma^{(3)}\left(\lambda^{(3)}\right)^{u_{1}} \lambda^{u_{2}} & \\
& =\left(\lambda^{(3)}-\lambda^{(2)}\right) \gamma \lambda^{u_{1}}\left(\lambda^{(2)}\right)^{u_{2}} .
\end{aligned}
$$

Dividing by $\left(\lambda^{(2)}-\lambda\right) \gamma^{(3)}\left(\lambda^{(3)}\right)^{u_{1}} \lambda^{u_{2}}$ gives

$$
\frac{\left(\lambda^{(3)}-\lambda\right) \gamma^{(2)}}{\left(\lambda^{(2)}-\lambda\right) \gamma^{(3)}}\left(\frac{\lambda^{(2)}}{\lambda^{(3)}}\right)^{u_{1}}\left(\frac{\lambda^{(3)}}{\lambda}\right)^{u_{2}}-1=\frac{\lambda^{(3)}-\lambda^{(2)}}{\lambda^{(2)}-\lambda} \cdot \frac{\gamma \lambda^{u_{1}}\left(\lambda^{(2)}\right)^{u_{2}}}{\gamma^{(3)}\left(\lambda^{(3)}\right)^{u_{1}} \lambda^{u_{2}}} .
$$


The right-hand side is non-zero and is also equal to

$$
\frac{\lambda^{(3)}-\lambda^{(2)}}{\lambda^{(2)}-\lambda} \cdot \frac{x-\lambda y}{x-\lambda^{(3)} y},
$$

thus, by (6) and (5), its absolute value is

$$
\leq\left|\frac{\lambda^{(3)}-\lambda^{(2)}}{\lambda^{(2)}-\lambda}\right| \frac{k}{\left(n^{2}+2\right) y^{3}} .
$$

We notice that

$$
\frac{\lambda^{(3)}-\lambda^{(2)}}{\lambda^{(2)}-\lambda}=\frac{\lambda^{(3)}+\frac{1}{\lambda^{(3)}+1}}{-\frac{\lambda^{(3)}+1}{\lambda^{(3)}}+\frac{1}{\lambda^{(3)}+1}}=-\lambda^{(3)} \cdot \frac{\left(\lambda^{(3)}\right)^{2}+\lambda^{(3)}+1}{\left(\lambda^{(3)}\right)^{2}+\lambda^{(3)}+1}=-\lambda^{(3)},
$$

hence, the considered right-hand side is $\leq k /\left(n y^{3}\right)$.

Now put

$$
\Lambda=u_{1} \log \left|\frac{\lambda^{(2)}}{\lambda^{(3)}}\right|+u_{2} \log \left|\frac{\lambda^{(3)}}{\lambda}\right|+\log \left|\frac{\gamma^{(2)}}{\gamma^{(3)}}\right|+\log \left|\frac{\lambda^{(3)}-\lambda}{\lambda^{(2)}-\lambda}\right| .
$$

Using the inequality $|\log z|<1.5 \cdot|z-1|$, which is true for $|z-1|<1 / 2$, we get

$$
0<|\Lambda|<3 k /\left(2 n y^{3}\right)
$$

The relation

$$
\frac{\lambda^{(3)}-\lambda}{\lambda^{(2)}-\lambda}=\frac{-1-1 / \lambda-\lambda}{-1 /(\lambda+1)-\lambda}=\lambda+1=-\frac{1}{\lambda^{(2)}}
$$

combined with $\lambda^{(3)} / \lambda=\left(\lambda^{(3)}\right)^{2} \lambda^{(2)}$ gives

$$
\Lambda=\left(u_{1}+u_{2}-1\right) \log \left|\lambda^{(2)}\right|+\left(2 u_{2}-u_{1}\right) \log \left|\lambda^{(3)}\right|+\log \left|\gamma^{(2)} / \gamma^{(3)}\right| .
$$

6. A first study of the linear form in logs. The previous linear form $\Lambda$ can be written as

$$
\Lambda=V l_{2}+v l_{3}+\log \left|\gamma^{(2)} / \gamma^{(3)}\right|,
$$

where we have put $V=u_{1}+u_{2}-1$ and $v=-u_{1}+2 u_{2}$. It satisfies

$$
|\Lambda|<3 k /\left(2 n y^{3}\right) .
$$

Grouping the last two terms we get a linear form of the type (we want to use the results of [LMN], so that we shall use the notations of this paper)

$$
\Lambda=b_{1} \log \alpha_{1}+b_{2} \log \alpha_{2},
$$

where $b_{1}=V, b_{2}=1, \alpha_{1}=\lambda^{(2)}$ and $\alpha_{2}=\left|\left(\lambda^{(3)}\right)^{v} \gamma^{(2)} / \gamma^{(3)}\right|$. Concerning $\alpha_{1}$, we have $|\log | \lambda^{(2)}|| \leq 1 / n$ and $M\left(\alpha_{1}\right)=\left|\lambda^{(2)} \lambda^{(3)}\right|$, which implies

$$
h\left(\alpha_{1}\right)=h(\lambda) \leq \frac{1}{3} \log (n+2) .
$$


By Corollary $2, \log \left(M\left(\gamma^{(2)} / \gamma^{(3)}\right)\right) \leq \log k+\frac{5}{2} \log (n+3)$. Thus,

$$
h\left(\alpha_{2}\right) \leq \frac{|v|}{3} \log (n+2)+\frac{1}{3}\left(\log k+\frac{5}{2} \log (n+3)\right) .
$$

For the convenience of the reader, we quote the main result of [LMN].

Theorem A. Let $K$ and $L$ be integers $\geq 3, R_{1}, S_{1}, R_{2}, S_{2}$ positive integers. Let $\varrho>1$ be a real number. Put $R=R_{1}+R_{2}-1, S=S_{1}+S_{2}-1$,

$$
g=\frac{1}{4}-\frac{K L}{12 R S}, \quad b=\left((R-1) b_{2}+(S-1) b_{1}\right)\left(\prod_{k=1}^{K-1} k !\right)^{-2 /\left(K^{2}-K\right)} .
$$

Let $a_{1}, a_{2}$ be real numbers such that

$$
a_{i} \geq \varrho\left|\log \alpha_{i}\right|-\log \left|\alpha_{i}\right|+2 D h\left(\alpha_{i}\right), \quad i=1,2,
$$

where $D=\left[\mathbb{Q}\left(\alpha_{1}, \alpha_{2}\right): \mathbb{Q}\right]$. Suppose that

$$
\begin{gathered}
\#\left\{\alpha_{1}^{r} \alpha_{2}^{s} ; 0 \leq r<R_{1}, 0 \leq s \leq S_{1}\right\} \geq L, \\
\#\left\{b_{1} r+s b_{2} ; 0 \leq r<R_{2}, 0 \leq s<S_{2}\right\}>(K-1) L
\end{gathered}
$$

and that

$$
\begin{array}{r}
K(L-1) \log \varrho+(K-1) \log 2-(D+1) \log (K L)-D(K-1) \log b \\
-g L\left(R a_{1}+S a_{2}\right)>0 .
\end{array}
$$

Then

$\left|\Lambda^{\prime}\right| \geq \varrho^{-K L+0.5}, \quad$ where $\Lambda^{\prime}=\Lambda \max \left\{\frac{L R e^{L R|\Lambda| /\left(2 b_{1}\right)}}{2 b_{1}}, \frac{L S e^{L S|\Lambda| /\left(2 b_{2}\right)}}{2 b_{2}}\right\}$.

In our case, we have $D=3$. The study above shows that one can take

$$
a_{1} \geq 2 \log (n+2)+(\varrho-1) / n
$$

and

$$
a_{2} \geq(2|v|+5) \log (n+3)+2 \log k+(\varrho-1) l_{0},
$$

where $l_{0}=\left|\log \alpha_{2}\right|$. For further reference, we notice that (by Corollary 2)

$$
l_{0} \leq\left(|v|+\frac{3}{2}\right) \log (n+3) .
$$

The algebraic numbers $\alpha_{1}$ and $\alpha_{2}$ are multiplicatively independent. In fact, assume that they are not. Then there exist non-zero integers $r$ and $s$ such that

$$
1=\alpha_{1}^{r} \alpha_{2}^{s}=\lambda^{(2) r} \lambda^{(3) s v}\left(\gamma^{(2)} / \gamma^{(3)}\right)^{s} .
$$

Thus $\gamma^{(2)} / \gamma^{(3)}$ is a unit in $\mathbb{Z}_{\mathbb{K}}$ and can be written as

$$
\gamma^{(2)} / \gamma^{(3)}=\lambda^{(2) t_{2}} \lambda^{(3) t_{3}}
$$

with suitable $t_{2}, t_{3} \in \mathbb{Z}$. Combining the last two equations and using the fact that $\lambda^{(2)}$ and $\lambda^{(3)}$ are multiplicatively independent we get $s\left(v+t_{3}\right)=0$ 
or $t_{3}=-v$. This implies

$$
\Lambda=\left(V+t_{2}\right) \log \left|\lambda^{(2)}\right|,
$$

and so either $\Lambda=0$ or $|\Lambda|>1 /(2 n)$. We already noticed that $\Lambda$ is non-zero, and the second alternative contradicts $|\Lambda|<3 k /\left(2 n y^{3}\right)<1 /(2 n y)$. The claim is proved.

Thus, in any case, the first one of conditions (7) holds if we suppose $R_{1} S_{1} \geq L$, and the choices of these two parameters will satisfy this condition.

To satisfy the second of conditions (7), we take

$$
R_{2}=\left[\sqrt{(K-1) L a_{2} / a_{1}}\right]+1, \quad S_{2}=\left[\sqrt{(K-1) L a_{1} / a_{2}}\right]+1,
$$

so that $R_{2} S_{2}>(K-1) L$.

Now, there are two possibilities: either the numbers $r b_{1}+s b_{2}, 0 \leq r<R_{2}$ and $0 \leq s<S_{2}$ are pairwise different and then the second of conditions (7) holds, or (since $b_{2}=1$ ) we have $|V| \leq S_{2}$.

We also take

$$
K=\left[\mu^{2} L a_{1} a_{2}\right]+1,
$$

where $0<\mu \leq 0.5$ and $L$ will be chosen later. Now we study the term $g\left(R a_{1}+S a_{2}\right)$. Since $R=R_{1}+R_{2}-1$ and $S=S_{1}+S_{2}-1$,

$$
\begin{aligned}
g\left(R a_{1}+S a_{2}\right)= & \left(\frac{1}{4}-\frac{K L}{12 R S}\right)\left(R a_{1}+S a_{2}\right) \\
\leq & \frac{1}{4}\left(\left(R_{2}-1\right) a_{1}+\left(S_{2}-1\right) a_{2}\right)+\frac{1}{4}\left(R_{1} a_{1}+S_{1} a_{2}\right) \\
& -\frac{K L}{12}\left(\frac{a_{1}}{\left(S_{2}-1\right)+S_{1}}+\frac{a_{2}}{\left(R_{2}-1\right)+R_{1}}\right) .
\end{aligned}
$$

Then, the definitions of $R_{2}$ and $S_{2}$ imply

$$
\begin{aligned}
g\left(R a_{1}+S a_{2}\right)= & \frac{1}{2} \sqrt{K L a_{1} a_{2}}+\frac{1}{4}\left(R_{1} a_{1}+S_{1} a_{2}\right) \\
& -\frac{K L}{12}\left(\frac{a_{1}}{\sqrt{K L a_{1} / a_{2}}+S_{1}}+\frac{a_{2}}{\sqrt{K L a_{2} / a_{1}}+R_{1}}\right) .
\end{aligned}
$$

Now we use the inequality

$$
\frac{a_{1}}{\sqrt{K L a_{1} / a_{2}}+S_{1}} \leq\left(\frac{a_{1}}{\sqrt{K L a_{1} / a_{2}}}\right)\left(1-\frac{S_{1}}{\sqrt{K L a_{1} / a_{2}}}\right)=\frac{\sqrt{a_{1} a_{2}}}{\sqrt{K L}}-\frac{a_{2} S_{1}}{K L},
$$

and the similar one with $R_{1}$, and get

$$
g\left(R a_{1}+S a_{2}\right) \leq \frac{1}{3} \sqrt{K L a_{1} a_{2}}+\frac{1}{3}\left(a_{1} R_{1}+a_{2} S_{1}\right) .
$$

Choosing $R_{1}=\left[\sqrt{L a_{2} / a_{1}}\right]+1$ and $S_{1}=\left[\sqrt{L a_{1} / a_{2}}\right]+1$ gives

$$
g\left(R a_{1}+S a_{2}\right) \leq \frac{1}{3} \sqrt{K L a_{1} a_{2}}+\frac{2 \sqrt{L a_{1} a_{2}}}{3}+\frac{a_{1}+a_{2}}{3} .
$$


By Lemma 9 of [LMN],

$$
b \leq \frac{(R-1) b_{2}+(S-1) b_{1}}{K-1} \exp \left\{\frac{3}{2}-\frac{\log (2 \pi(K-1) / \sqrt{e})}{K-1}+\frac{\log K}{6 K(K-1)}\right\},
$$

where

$$
\begin{aligned}
(R-1) b_{2}+(S-1) b_{1} \leq & \left(\sqrt{(K-1) L a_{2} / a_{1}}+\sqrt{L a_{2} / a_{1}}+1\right) \\
& +\left(\sqrt{(K-1) L a_{1} / a_{2}}+\sqrt{L a_{1} / a_{2}}+1\right) V .
\end{aligned}
$$

These remarks show that condition (8) holds if

$$
\begin{aligned}
& K(L-1) \log \varrho+(K-1) \log 2-4 \log (K L)-3(K-1) H_{0}-\frac{3}{2}(K-1) \\
& +\log (\pi(K-1) / \sqrt{e})-\frac{\log K}{6 K}-\frac{L}{3} \sqrt{K L a_{1} a_{2}}-\frac{2 L^{3 / 2} a_{1} a_{2}}{3}-\frac{L\left(a_{1}+a_{2}\right)}{3} \geq 0,
\end{aligned}
$$

where we have put

$$
H_{0}=\log \left(\frac{\sqrt{L a_{2} / a_{1}}(\sqrt{K-1}+1)+1+\left(\sqrt{L a_{1} / a_{2}}(\sqrt{K-1}+1)+1\right) V}{K-1}\right) .
$$

We have

$$
\begin{aligned}
& \frac{\sqrt{L a_{2} / a_{1}}(\sqrt{K-1}+1)+1+\left(\sqrt{L a_{1} / a_{2}}(\sqrt{K-1}+1)+1\right) V}{K-1} \\
& \quad \leq \frac{K}{K-1}\left((1+1 / \sqrt{K}) \frac{\sqrt{L a_{2} / a_{1}}+V \sqrt{L a_{1} / a_{2}}}{\sqrt{K}}+\frac{V+1}{K}\right) \\
& \leq \frac{K}{K-1}\left(\left(1+\frac{1}{\sqrt{K}}\right) \frac{1}{\mu}\left(\frac{1}{a_{1}}+\frac{V}{a_{2}}\right)+\frac{V+1}{\mu^{2} L a_{1} a_{2}}\right) \\
& \leq \frac{K}{K-1} \cdot \frac{1}{\mu}\left(\frac{1}{a_{1}}+\frac{V}{a_{2}}\right)\left(1+\frac{1}{\sqrt{K}}+\frac{1}{\mu L}\left(\frac{1}{a_{1}}+\frac{1}{a_{2}}\right)\right) .
\end{aligned}
$$

We put $\theta=\log \varrho$. Choosing $\mu=2 /(3 \theta)$, and using the definition $K=$ $\left[\mu^{2} L a_{1} a_{2}\right]+1$, we get

$$
\begin{aligned}
H_{0} \leq H:= & \log \left(\frac{K}{K-1}\right)+\log \left(1+\frac{1}{\sqrt{K}}+\frac{3 \theta}{2 L}\left(\frac{1}{a_{1}}+\frac{1}{a_{2}}\right)\right) \\
& +\log ^{+}\left(\frac{1}{\mu a_{1}}+\frac{V}{\mu a_{2}}\right),
\end{aligned}
$$

where, as usual, $\log ^{+} x=\max \{0, \log x\}$. Then we see that condition (8) is satisfied whenever

$$
\begin{aligned}
\Phi:=\frac{2}{3 \theta}\left(\frac{2(L-1)}{3}-\frac{2 H}{\theta}-\frac{L}{3}+\frac{2 \log 2}{3 \theta}\right) & -\frac{7 \log L}{a_{1} a_{2} L}-\frac{3 \log \left(4 a_{1} a_{2} /\left(9 \theta^{2}\right)\right)}{a_{1} a_{2} L} \\
& -\frac{2 \sqrt{L}}{3 \sqrt{a_{1} a_{2}}}-\frac{1}{3}\left(\frac{1}{a_{1}}+\frac{1}{a_{2}}\right) \geq 0 .
\end{aligned}
$$


[We divided by $\left.L a_{1} a_{2}.\right]$ Now, we choose $L=\max \{16,[\varepsilon+6 H / \theta]+3\}$, where $\varepsilon>0$ is to be chosen later. This choice implies

$$
\begin{aligned}
\Phi \geq & \frac{4 \log 2}{9 \theta^{2}}-\frac{7 \log L}{a_{1} a_{2} L}-\frac{3 \log \left(4 a_{1} a_{2} /\left(9 \theta^{2}\right)\right)}{a_{1} a_{2} L}-\frac{1}{3}\left(\frac{1}{a_{1}}+\frac{1}{a_{2}}\right) \\
& +\frac{4 \varepsilon L}{9 \theta}-\frac{2 \sqrt{L}}{3 \sqrt{a_{1} a_{2}}} \\
\geq & \frac{4 \log 2}{9 \theta^{2}}-\frac{1.22}{a_{1} a_{2}}-\frac{3 \log \left(4 a_{1} a_{2} /\left(9 \theta^{2}\right)\right)}{16 a_{1} a_{2}}-\frac{1}{3}\left(\frac{1}{a_{1}}+\frac{1}{a_{2}}\right) \\
& +\sqrt{L}\left(\frac{4 \varepsilon \sqrt{L}}{9 \theta}-\frac{2}{3 \sqrt{a_{1} a_{2}}}\right) .
\end{aligned}
$$

Thus, $\Phi \geq 0$, when both

$$
\Theta:=\frac{4 \log 2}{9 \theta^{2}}-\frac{1.22}{a_{1} a_{2}}-\frac{3 \log \left(4 a_{1} a_{2} /\left(9 \theta^{2}\right)\right)}{16 a_{1} a_{2}}-\frac{1}{3}\left(\frac{1}{a_{1}}+\frac{1}{a_{2}}\right) \geq 0
$$

and

$$
\varepsilon \geq \frac{3 \theta}{8 \sqrt{a_{1} a_{2}}}
$$

We take $\varrho=15$, and in this case $\Theta \geq 0$ for

$$
a_{2} \geq a_{1} \geq 15, \quad a_{2} \geq 24, \quad \varepsilon=0.054 .
$$

Then (8) holds, and we get

$$
\log \left|\Lambda^{\prime}\right| \geq-\left(\mu^{2} L^{2} a_{1} a_{2}-L\right) \theta=-\frac{4}{9 \theta} L^{2} a_{1} a_{2}-L \theta .
$$

The choices of $R$ and $S$ imply $\max \{R, S\} \leq L \max \left\{a_{1}, a_{2}\right\}$, and the definition of $\Lambda^{\prime}$ leads to

$$
\log \left|\Lambda^{\prime}\right| \leq \log |\Lambda|+2 \log L+\log \left(\max \left\{a_{1}, a_{2}\right\}\right),
$$

except maybe when $|\Lambda| \geq 1 /\left(L^{2} \max \left\{a_{1}, a_{2}\right\}\right)$. Hence, we always have

$$
\begin{aligned}
\log |\Lambda| & \geq-\frac{4}{9 \theta} L^{2} a_{1} a_{2}-L \theta-2 \log L-\log \left(\max \left\{a_{1}, a_{2}\right\}\right) \\
& \geq-\left(\frac{4}{9 \theta} L^{2}+\frac{0.01}{a_{1}}\right) a_{1} a_{2}-(\theta+0.35) L .
\end{aligned}
$$

Recall that

$$
\log |\Lambda| \leq \log \frac{3 k}{2 n}-3 \log y .
$$

Thus, under condition (9), we get

$$
\log y \leq \frac{1}{3}\left(\frac{4}{9 \theta}+\frac{0.01}{a_{1}}\right) L^{2} a_{1} a_{2}+\frac{\theta+0.35}{3} L+\frac{1}{3} \log \frac{3 k}{2 n} .
$$


7. A second study of linear forms and the proof of Theorem 1. Applying conjugations to relation (3) and taking logs, we get the system of relations

where

$$
\begin{aligned}
& u_{1} l_{2}+u_{2} l_{3}=\log y+\eta_{2}, \\
& u_{1} l_{3}+u_{2} l_{1}=\log y+\eta_{3},
\end{aligned}
$$

$$
\eta_{2}=\log \left|x / y-\lambda^{(2)}\right|-\log \left|\gamma^{(2)}\right| \quad \text { and } \quad \eta_{3}=\log \left|x / y-\lambda^{(3)}\right|-\log \left|\gamma^{(3)}\right| .
$$

By (5), $0 \leq \log \left|x / y-\lambda^{(2)}\right| \leq 2 / n^{2}$, and $\log n \leq \log \left|x / y-\lambda^{(3)}\right| \leq \log (n+1)$. Moreover, the choice of $\gamma$ implies

$$
\frac{k^{1 / 3}}{\sqrt{n+3}} \leq\left|\gamma^{(2)}\right| \leq k^{1 / 3} \sqrt{n+3}, \quad \frac{k^{1 / 3}}{n+3} \leq\left|\gamma^{(3)}\right| \leq k^{1 / 3}(n+3) .
$$

These estimates lead to

$$
-\frac{1}{2} \log (n+3)-\frac{1}{3} \log k \leq \eta_{2} \leq \frac{2}{n^{2}}-\frac{1}{3} \log k+\frac{1}{2} \log (n+3)
$$

and

$$
-\log (n+3)+\log n-\frac{1}{3} \log k \leq \eta_{3} \leq-\frac{1}{3} \log k+2 \log (n+3) .
$$

The above system implies

$$
\begin{aligned}
& u_{1}\left(l_{3}^{2}-l_{1} l_{2}\right)=\left(l_{3}-l_{1}\right) \log y-l_{1} \eta_{2}+l_{3} \eta_{3}, \\
& u_{2}\left(l_{3}^{2}-l_{1} l_{2}\right)=\left(l_{3}-l_{2}\right) \log y+l_{3} \eta_{2}-l_{2} \eta_{3} .
\end{aligned}
$$

Hence,

$$
V=u_{1}+u_{2}-1=\frac{1}{l_{3}^{2}-l_{1} l_{2}}\left(\left(2 l_{3}-l_{1}-l_{2}\right) \log y+\left(l_{3}-l_{1}\right) \eta_{2}+\left(l_{3}-l_{2}\right) \eta_{3}\right)-1
$$

Put $\Delta=l_{3}^{2}-l_{1} l_{2}$. Then

$$
\Delta(V+1)-\left(2 l_{3}-l_{1}-l_{2}\right) \log y=\left(l_{3}-l_{1}\right) \eta_{2}+\left(l_{3}-l_{2}\right) \eta_{3} .
$$

Using the estimates of the $l_{i}$ 's (end of Section 3) and those of $\eta_{2}$ and $\eta_{3}$, we get

$$
\begin{aligned}
\mid \Delta(V & +1)-\left(2 l_{3}-l_{1}-l_{2}\right) \log \left(y / k^{1 / 3}\right)-\left(l_{3}-l_{2}\right) \log (n+3) \mid \\
& \leq\left(\left(l_{3}-l_{2}\right) \log \left((n+3)^{2} / n\right)+\left(l_{3}-l_{1}\right) \log (n+3)\right) \\
& \leq\left(\left(\log n+\frac{2}{n^{2}}\right) \log \left((n+3)^{2} / n\right)+2\left(\log (n+1)+\frac{2}{n^{2}}\right) \log (n+3)\right) \\
& \leq 3 \log (n+2) \log (n+3)
\end{aligned}
$$

for $n \geq 5$. Notice that

$$
\Delta \geq \log ^{2} n+\frac{\log (n+1)}{n+1}>\log ^{2} n
$$


and

$2 l_{3}-l_{1}-l_{2}<2 \log n+\frac{4}{n^{2}}+\log (n+1)+\frac{2}{n(n+1)}-\frac{n-1 / 2}{n^{2}+2} \leq 3 \log (n+1)$,

and also

$$
2 l_{3}-l_{1}-l_{2}>2 \log n+\frac{4}{(n+1)^{2}}+\log (n+1)+\frac{2}{(n+1)^{2}}-\frac{1}{n}>3 \log n,
$$

for $n \geq 3$

We notice that this implies $V \geq 0$ for $n \geq 5$ and

$$
\begin{aligned}
V+1 & \leq \frac{1}{\Delta}\left(3 \log (n+1) \log \frac{y}{k^{1 / 3}}+4 \log (n+2) \log (n+3)\right) \\
& \leq \frac{\log (n+2)}{\log ^{2} n}\left(3 \log \left(y / k^{1 / 3}\right)+4 \log (n+3)\right),
\end{aligned}
$$

for $n \geq 5$.

After this list of estimates, let us bound $V$. Recall that

$$
\log y \leq \frac{1}{3}\left(\frac{4}{9 \theta}+0.01\right) L^{2} a_{1} a_{2}+\frac{\theta+0.35}{3} L+\frac{1}{3} \log \frac{3 k}{2 n} .
$$

By the conditions on $a_{1}$ and $a_{2}$, and since $\varrho=15$ and $L \geq 16$ this implies

$$
\log y \leq \frac{1}{3} 0.17423 \cdot L^{2} a_{1} a_{2}+\frac{1}{3} \log \frac{3 k}{2 n} .
$$

Since $a_{1} \geq 2 \log (n+2)$, we get (for $n \geq 5$ )

$$
\begin{aligned}
V+1 & \leq \frac{\log (n+2)}{\log ^{2} n}\left(0.17423 \cdot L^{2} a_{1} a_{2}+4 \log (n+3)\right) \\
& \leq \frac{\log (n+2)}{\log ^{2} n} \cdot 0.17456 \cdot L^{2} a_{1} a_{2} .
\end{aligned}
$$

We consider the two cases

$$
L=\left\{\begin{array}{l}
16, \\
{[6 H / \theta+\varepsilon]+3 \geq 17 .}
\end{array}\right.
$$

In the first case,

$$
V+1 \leq \frac{\log (n+2)}{\log ^{2} n} \cdot 44.688 \cdot a_{1} a_{2} .
$$

Now suppose $L \geq 17$. The definition of $H$ implies $H \leq 1.522+\log ^{+}\left(|V| / a_{2}\right)$, and the definition of $L$, combined with $L \geq 17$, implies $\log \left(V / a_{2}\right) \geq 4.77$. 
Then the inequality

$$
\begin{aligned}
\frac{V}{a_{2}} & \leq \frac{\log (n+2)}{\log ^{2} n} \cdot 0.17456 \cdot L^{2} a_{1} \\
& \leq 0.17456 \cdot \frac{a_{1} \log (n+2)}{\log ^{2} n}\left[3.054+\frac{6\left(1.522+\log \left(V / a_{2}\right)\right)}{\theta}\right]^{2}
\end{aligned}
$$

leads to a contradiction. Thus we have proved that

$$
V+1 \leq \frac{\log (n+2)}{\log ^{2} n} \cdot 44.688 \cdot a_{1} a_{2} \quad \text { and } \quad L=16 .
$$

We have just obtained an upper bound for $|V|$, using a non-trivial lower bound of $\Lambda$. Using again $\Lambda$, we directly get a lower bound for $|V|$. Indeed, $\Lambda=V l_{2} \pm l_{0}$, where $\left|l_{2}\right|<(n+1) /\left(n^{2}+n+2\right)$; since $|\Lambda|$ is very small, this implies

$$
V \geq n l_{0} .
$$

Suppose that $n \geq 1650$, then (in this case $V \geq 0$ )

$$
V+1 \leq \frac{\log (n+2)}{\log ^{2} n} \cdot 44.688 \cdot a_{1} a_{2} \leq \frac{44.696}{\log n} \cdot a_{1} a_{2},
$$

where

$$
\begin{aligned}
& a_{1}=\max \{2 \log (n+2)+14 / n, 15\}<2.03 \log n, \\
& a_{2}=\max \left\{\left(2|v|+\frac{5}{2}\right) \log (n+3)+\log k+14 l_{0}, 24\right\} .
\end{aligned}
$$

Combining these estimates gives

$$
V<89.5 a_{2} \text { for } n \geq 1650 .
$$

If $v \neq 0$ then $a_{2}=\left(2|v|+\frac{5}{2}\right) \log (n+3)+\log k+14 l_{0}$, and

$$
n l_{0}<89.5\left(\left(2|v|+\frac{5}{2}\right) \log (n+3)+\log k+14 l_{0}\right) .
$$

Hence, if $v \neq 0$,

$$
(n-1253) l_{0}<(179|v|+223.75) \log (n+3)+89.5 \log k
$$

for $n \geq 1650$. Since

$$
l_{0} \geq|v| \log n-\frac{3}{2} \log (n+3)>(|v|-1.501) \log n,
$$

for $n \geq 1650$, we get also

$$
(n-1253-179.1)|v|<(1.501(n-1253)+223.75)+89.5 \frac{\log k}{\log n},
$$

or

$$
|v|<\left(1-\frac{1432.1}{n}\right)^{-1}\left\{1.501-\frac{1657}{n}+89.5 \frac{\log k}{n \log n}\right\}
$$


Now we consider the $y$ term and we suppose $n \geq 1650$. We have

$$
\log y \leq \frac{1}{3} 0.17423 \cdot L^{2} a_{1} a_{2}+\frac{1}{3} \log \frac{3 k}{2 n},
$$

with $a_{1}<2.03 \log n$ and $L=16$, hence

$$
\log y \leq 29.77 a_{2} \log n+\frac{1}{3} \log k .
$$

Using the inequality $l_{0} \leq|v| \log (n+1)+1.5 \log (n+3)$ and the definition of $a_{2}$, we get

$$
\log y<29.77(16|v|+23.5) \log ^{2}(n+2)+29.82 \log n \cdot \log k .
$$

And the upper bound on $|v|$ gives

$$
\begin{aligned}
\log y< & \left(700+476.4\left(1-\frac{1432.1}{n}\right)^{-1}\left\{1.501-\frac{1902}{n}\right\}\right) \log ^{2}(n+2) \\
& +\left(29.82+\left(1-\frac{1432.1}{n}\right)^{-1} \frac{1432}{n \log n}\right) \log n \cdot \log k .
\end{aligned}
$$

Theorem 1 is proved.

8. An improved bound if $k$ is small. In this section we add some hypothesis on $k$ and then get an upper bound for $l_{0}$, which leads to upper bounds on $|v|$ and $y$.

We suppose $n \geq 1650$ and $k \leq n^{4}$. Then the upper bound on $v$ becomes

$$
|v|<\left(1-\frac{1432.1}{n}\right)^{-1}\left\{1.501-\frac{1657}{n}+\frac{358}{n}\right\}
$$

which implies

$$
|v| \leq 1 \quad \text { for } n \geq 2750 \text {. }
$$

Using the definition of $a_{2}$, we also obtain

$$
l_{0}<\max \left\{\frac{761 \log n}{n-1253}, \frac{2148}{n}\right\}=\frac{761 \log n}{n-1253} \quad \text { for } n \geq 3150 .
$$

This implies

$$
l_{0}<\frac{\log n}{n^{1 / 3}} \quad \text { for } n \geq 23500 .
$$

In this case, we choose $\varrho=n^{1 / 3}$; then $\mu=2 / \log n$. Since $a_{1}>2 \log n$ and $a_{2}>2.5 \log n$, taking again $L=16$, we have $K \geq 360$. It is also easy to verify that $H<4.67+\log \log n$ and that we can take $\varepsilon=0.17$ (the number $\varepsilon$ occurs in the definition of the integer $L$ ). These estimates prove that the choice $L=16$ is legitimate. Then applying again Theorem A, for $n \geq 23500$, 
we get

$$
\begin{aligned}
\log y & \leq \frac{1}{3}\left(\frac{4}{9 \theta}+\frac{0.01}{a_{1}}\right) L^{2} a_{1} a_{2}+\frac{\theta+0.35}{3} L+\frac{1}{3} \log \frac{3 k}{2 n} \\
& \leq \frac{1}{3}\left(\frac{4}{3}+0.005\right) 256 \cdot 2.02 \cdot 9.5 \cdot \log n+\frac{\theta+0.35}{3} L+\frac{1}{3} \log \frac{3 k}{2 n} .
\end{aligned}
$$

Thus, we have proved that

$$
\log y<2180 \log n \quad \text { if } n \geq 23500 \text { and } k \leq n^{4} .
$$

9. Proof of Theorem 3. Let us consider the inequality

$$
\left|x^{3}-(n-1) x^{2} y-(n+2) x y^{2}-y^{3}\right| \leq k
$$

with $k=2 n+1$. The family of equations $\left|F_{n}(x, y)\right|=1$ was completely solved by Thomas [T1] and Mignotte [M]. Moreover, it was proved by Lemmermeyer and Pethő [LP] that if $\gamma \in \mathbb{Z}[\lambda]$ has norm, in absolute value, less than $2 n+1$, then $\gamma$ is a rational integer multiple of a binomial unit from $\mathbb{Z}[\lambda]$. Thus to prove Theorem 3 it remains to study the equation

$$
\left|x^{3}-(n-1) x^{2} y-(n+2) x y^{2}-y^{3}\right|=2 n+1 .
$$

It is easy to see that

$$
\begin{aligned}
F_{n}(1,1) & =F_{n}(1,-2)=F_{n}(1,-n-1)=F_{n}(n, 1) \\
& =F_{n}(-n-1, n)=F_{n}(-2,1)=-(2 n+1),
\end{aligned}
$$

and

$$
\begin{aligned}
F_{n}(-1,-1) & =F_{n}(-1,2)=F_{n}(-1, n+1) \\
& =F_{n}(-n,-1)=F_{n}(n+1,-n)=F_{n}(2,-1)=2 n+1 .
\end{aligned}
$$

The continued fraction expansion of $\lambda$ is $\lambda=[-1,1, n,[n / 2], \ldots]$ if $n>0$, and hence its first convergents are

$$
-1,0,-\frac{1}{n+1}, \frac{-[n / 2]}{(n+1)[n / 2]+1} .
$$

Let $(x, y) \in \mathbb{Z}^{2}$ be a solution of (10) such that $y \geq 1$ and

$$
|\lambda y-x|<\left|\lambda^{(2)} y-x\right|,\left|\lambda^{(3)} y-x\right| .
$$

By Corollary 1 , and by $D_{f}=\left(n^{2}+n+7\right)^{2}$ we have

$$
\left|L_{1}\right| \leq\left(\frac{4(2 n+1)^{4}}{\left(n^{2}+n+7\right)^{2}}\right)^{1 / 6} \frac{1}{y^{2}}<\frac{2}{y^{2}}
$$

thus $|x / y-\lambda|<2 / 25$ if $|y| \geq 5$. Using the estimations for $\lambda, \lambda^{(2)}$ and $\lambda^{(3)}$ we get

$$
\left|x-\lambda^{(2)} y\right|>\left(\left|\lambda^{(2)}-\lambda\right|-\frac{2}{25}\right)|y|>\left(1-\frac{2}{25}\right)|y|=\frac{23}{25}|y|
$$


and

Therefore

$$
\left|x-\lambda^{(3)} y\right|>\left(\left|\lambda^{(3)}-\lambda\right|-\frac{2}{25}\right)|y|>\left(n-\frac{2}{25}\right)|y| .
$$

$$
\left|\frac{x}{y}-\lambda\right|<\frac{2 n+1}{\frac{23}{25}\left(n-\frac{2}{25}\right)|y|^{3}}<\frac{1}{2 y^{2}}
$$

if $|y| \geq 5$ and $n \geq 3$, which means that in those cases $x / y$ is a convergent of $\lambda$. We conclude the same for $n=1$ when $|y| \geq 8$ and for $n=2$ when $|y| \geq 6$.

If $n=0$, then (10) is already solved (see Thomas [T1] and Mignotte $[\mathrm{M}])$. For $n=1$ with $|y| \leq 7$ and $n=2$ with $|y| \leq 5$ we get by a direct computation the following solutions.

Table 1

\begin{tabular}{c|rrrrrrr}
\hline$n$ & 1 & 2 & 2 & 2 & 2 & 2 & 2 \\
\hline$x$ & -1 & -1 & -3 & -4 & -1 & 8 & -1 \\
$y$ & 2 & 2 & 2 & 3 & 3 & 3 & 4 \\
$f_{n}(x, y)$ & 3 & 5 & -5 & 5 & 5 & 5 & -5 \\
\hline
\end{tabular}

We remark that exactly the values given in the table are the non-trivial solutions for $n=2$.

If $n \geq 3$ and $4 \geq|y| \geq 2$ then $|\lambda|<2 / 9$ and by using $|x-\lambda y|<2 /|y|$ we get

$$
-2<-\frac{2}{|y|}-|\lambda| \cdot|y|<|x|<\frac{2}{|y|}+|\lambda| \cdot|y|<2,
$$

but the solutions with $|x| \leq 1$ are listed at the beginning. We did the same for $|y| \leq 1$. Thus apart from the cases listed in Table 1, the solutions of (10) come from convergents of $\lambda$, hence $|y| \geq n+1$. If $y=n+1$, then

$$
F_{n}(x, n+1)-(2 n+1)=(x+1)\left(x^{2}-n^{2} x-\left(n^{3}+3 n^{2}+5 n+2\right)\right) .
$$

If the second factor $q(x)$ is reducible over $\mathbb{Q}[x]$, then it has an integer zero. But a simple calculation shows that $q(-n-1)<0, q(-n-3)>0$ and $q(-n-2)=0$ iff $n=2$. Thus $q(x)$ is reducible over $\mathbb{Q}[x]$ iff $n=2$ and these cases are listed in Table 1.

Let $q_{1}(x)=F_{n}(x, n+1)+2 n+1$. Then by $q_{1}(0)<0, q_{1}(-1)>0$, $q_{1}(-n-2)=n^{2}+3 n>0$, and

$$
\begin{aligned}
q_{1}(-n-3) & =-n^{3}-3 n^{2}-5 n-12<0, \\
q_{1}\left(n^{2}+n+1\right) & =-\left(n^{4}+4 n^{3}+6 n^{2}+5 n+1\right), \\
q_{1}\left(n^{2}+n+2\right) & =-n^{3}+n^{2}+n+7, \\
q_{1}\left(n^{2}+n+3\right) & =n^{4}+2 n^{3}+12 n^{2}+13 n+29
\end{aligned}
$$


we see that $q_{1}(x)$ has no integer zero if $n \geq 0$. Thus we may assume $y>n+1$, whence the continued fraction expansion of $\lambda$ implies $y \geq(n+1)[n / 2]+1>$ $n^{2} / 2$.

By Theorem 3.1 of Lemmermeyer and Pethő $[\mathrm{LP}]$, all elements of $\mathbb{Z}[\lambda]$ of norm $2 n+1$ are associated with one of the conjugates of $\lambda-1$. It is easy to see that $\lambda^{(2)}-1=-(\lambda+2) /(\lambda+1)$ and $\lambda^{(3)}-1=-(2 \lambda+1) / \lambda$ and that $\lambda-1, \lambda+2$ and $2 \lambda+1$ satisfy the conclusion of Corollary 2 . Hence we may apply the results of Sections 6 and 7 .

First we prove that if $n \geq 1700$ then (10) has only the trivial solutions. We distinguish three cases and adopt the notations of Section 6 . We recall that if $n \geq 5$ then $V \geq 0$ and so $v \leq 1$.

Case I: $\gamma=\gamma_{1}=\lambda-1$. Now we have

$$
\alpha_{2}=\lambda^{(3) v}\left|\frac{\lambda^{(2)}-1}{\lambda^{(3)}-1}\right|, \quad h\left(\alpha_{2}\right) \leq \frac{1}{3}(|v| \log (n+2)+\log (n(2 n+1))) .
$$

By the choice $\varrho=15$ we also have $a_{2}=14 l_{0}+2(|v|+2) \log (n+2)+2 \log 2$, where

$$
|v-1| \log \lambda^{(3)}+\log 2+\frac{1}{2(n-1)} \leq l_{0} \leq|v-1| \log \lambda^{(3)}+\log 2+\frac{3}{2(n-1)} .
$$

As $V \geq 0$ and $v \leq 1$ we have $V>n l_{0}>n(|v-1| \log n+\log 2)$. On the other hand,

$$
V<89.5 a_{2} \leq 1253 l_{0}+179(|v|+2) \log (n+2)+179 \log 2 .
$$

Combining these inequalities we get $n<8450$ for $v=1$ and $n<1620$ for $v<1$.

Case II: $\gamma=\gamma_{2}=\lambda+2$. We now transform

$$
\alpha_{2}=\lambda^{(3) v}\left|\frac{\lambda^{(2)}+2}{\lambda^{(3)}+2}\right|
$$

to a more appropriate algebraic number to prove a sharp bound for $n$. Indeed, using $\lambda^{(2)}+2=(\lambda-n) \lambda$ and $\lambda^{(3)}+2=\left(\lambda^{(2)}-n\right) \lambda^{(2)}$ we get

$$
\frac{\lambda^{(2)}+2}{\lambda^{(3)}+2}=\frac{(\lambda-n) \lambda(\lambda+1)^{2}}{n(\lambda+1)+1}=\frac{n-\lambda}{n(\lambda+1)+1} \cdot \frac{1}{\lambda^{(3)} \lambda^{(2) 3}} .
$$

Thus our linear form becomes

$$
\Lambda=(V-3) \log \left|\lambda^{(2)}\right|+(v-1) \log \left|\lambda^{(3)}\right|+\log \left|\frac{n-\lambda}{n(\lambda+1)+1}\right| .
$$

By the estimates of $\lambda$, we also have

$$
1-\frac{2}{n^{3}}<\frac{n-\lambda}{n+n \lambda+1}=1-\frac{(n+1) \lambda+1}{n(\lambda+1)+1}<1-\frac{1}{n^{3}} .
$$


We proved $|\Lambda|<3 k /\left(2 n y^{3}\right)$ in Section 5 for all $n \geq 3$ and $k \in \mathbb{N}$. With our special value $k=2 n+1$ using the estimate $y>n^{2} / 2$ we get

$$
|\Lambda|<\frac{12(2 n+1)}{n^{7}}<\frac{1}{n^{3}}
$$

if $n \geq 4$ immediately. This inequality also remains true for $n=3$, which one can verify by a direct computation. Hence $v=1$ is impossible and if $v<1$ then

$|(V-3) \log | \lambda^{(2)}||>|v-1| \log \left|\lambda^{(3)}\right|-|\Lambda|-\left|\log \frac{n-\lambda}{n+n \lambda+1}\right|>|v-1| \log n$,

which implies $V-3>|v-1| n \log n$. On the other hand, $V<89.5 a_{2}$ with $a_{2}=(16|v|+18) \log (n+2)+2 \log 2$. These inequalities imply again $n<1620$.

Case III: $\gamma=\gamma_{3}=2 \lambda+1$. This case is similar to Case I, and therefore we have given only the most important data:

$$
\alpha_{2}=\lambda^{(3) v}\left|\frac{2 \lambda^{(2)}+1}{2 \lambda^{(3)}+1}\right|, \quad h\left(\alpha_{2}\right) \leq \frac{1}{3}((|v|+2) \log (n+2)+2 \log 2)
$$

and

$$
|v-1| \log n-\log 2<l_{0}<|v-1| \log (n+2)-\log 2 .
$$

Thus $a_{2}<(16|v|+18) \log (n+2)-10 \log 2$. If $v=1$, then $n<8640$ and if $v<1$, then $n<1700$.

We have proved that equation (10) has just the "trivial solutions" for $n>8640$; moreover, $v=1$ if $1700 \leq n \leq 8640$ and this can occur only in Cases I and III. We shall prove now the impossibility of $v=1$.

From $|\Lambda|<12(2 n+1) / n^{7}$ we get

$$
\left|V+v \frac{\log \left|\lambda^{(3)}\right|}{\log \left|\lambda^{(2)}\right|}+\frac{\log \left|\gamma_{i}^{(2)}\right|-\log \left|\gamma_{i}^{(3)}\right|}{\log \left|\lambda^{(2)}\right|}\right|<\frac{1}{n^{5}}
$$

for $n \geq 30$ and $i=1,2,3$. Putting

$$
\delta_{1}=\frac{\log \left|\lambda^{(3)}\right|}{\log \left|\lambda^{(2)}\right|} \quad \text { and } \quad \delta_{2 i}=\frac{\log \left|\gamma_{i}^{(2)}\right|-\log \left|\gamma_{i}^{(3)}\right|}{\log \left|\lambda^{(2)}\right|}
$$

this can be reformulated to

$$
\left\|v \delta_{1}+\delta_{2 i}\right\|<1 / n^{5},
$$

$i=1,2$ or 3 , where \|\| denotes the distance to the nearest integer.

For $1700 \leq n \leq 8640$ and $i=1$ and 3 we tested (11) with $v=1$, but did not found any $n$ satisfying (11) even with the much larger right hand side $10^{-5}$. We performed the same test with $v=1$ for the values $1 \leq i \leq 3$ and $0 \leq n<1700$ with the same result. Thus it remains to prove Theorem 3 only in the range $0 \leq n \leq 1700$ and with $v \neq 1$. 
10. The case $0 \leq n \leq 1700$ and end of the proof of Theorem 3 . In the range $0 \leq n \leq 1700$ application of linear forms in two logarithms fails to work because we are not able to prove an upper bound for $|v|$ and $V$. Therefore we apply the following more general theorem of Baker and Wüstholz $[\mathrm{BW}]$ :

TheOREM B. For a linear form $\Lambda \neq 0$ in logarithms of $m$ real algebraic numbers $\alpha_{1}, \ldots, \alpha_{m}$ with rational integer coefficients $b_{1}, \ldots, b_{m}$ we have

$$
\log |\Lambda| \geq-18(m+1) ! m^{m+1}(32 D)^{m+2} h\left(\alpha_{1}\right) \ldots h\left(\alpha_{m}\right) \log B,
$$

where $B=\max \left\{\left|b_{1}\right|, \ldots,\left|b_{m}\right|\right\}$, and where $D$ is the degree of the number field generated by $\alpha_{1}, \ldots, \alpha_{m}$.

In the present case we have $m=3, D=3, B=V$,

$$
\begin{gathered}
h\left(\lambda^{(2)}\right)=h\left(\lambda^{(3)}\right) \leq \frac{1}{3} \log (n+2), \\
h\left(\gamma_{i}^{(2)} / \gamma_{i}^{(3)}\right) \leq \frac{1}{3} \log (n(2 n+1)), \quad i=1,2,3,
\end{gathered}
$$

and we get

$$
\begin{aligned}
\log |\Lambda| & \geq-18 \cdot 4 ! \cdot 3^{6} \cdot 32^{5} \cdot \log ^{2}(n+2) \log (n(2 n+1)) \log V \\
& >-1.06 \cdot 10^{13} \cdot \log ^{2}(n+2) \log (n(2 n+1)) \log V .
\end{aligned}
$$

On the other hand, if $|y| \geq 2$ and $n \geq 5$ then

$$
\log |\Lambda|<-(V+1) \frac{\log ^{2} n}{\log (n+2)}+4 \log (n+3)-\log n+\log \frac{3}{2} .
$$

A comparison of these estimates leads to $V<10^{17}$.

This bound is much worse than that we got for $n>1650$ and demonstrates that the estimates for linear forms in two logarithms are much more suitable for solving diophantine problems completely than the general estimates. Unfortunately, estimates for two logs are not always applicable.

Despite the large bound for $V$ we can prove that our problem has no nontrivial solution also in the present range. For this purpose we use a variant of the Baker-Davenport lemma [BD], which was also helpful in [MPR]. We recall that

$$
\begin{aligned}
\left|\Lambda_{i}\right| & =\left|V+v \delta_{1}+\delta_{2 i}\right|<\frac{3(2 n+1)}{2 n y^{3} \log \left|\lambda^{(2)}\right|} \\
& <\exp \left\{-(V+1) \frac{\log ^{2} n}{\log (n+2)}+4 \log (n+3)+\log 3\right\}
\end{aligned}
$$

$(i=1,2,3)$, where the numbers $\delta_{1}$ and $\delta_{2 i}$ were defined in the previous section. 
Lemma 4. Suppose that $n \geq 5,1 \leq i \leq 3, V<10^{17}, v \neq 1$ and $\left|\Lambda_{i}\right|<10^{-50}$ and that $\widetilde{\delta}_{1}$ and $\widetilde{\delta}_{2 i}$ are rational numbers such that

$$
\left|\delta_{1}-\widetilde{\delta}_{1}\right|<10^{-50}, \quad\left|\delta_{2 i}-\widetilde{\delta}_{2 i}\right|<10^{-50} .
$$

If there exists a convergent $p / q$ in the continued fraction expansion of $\widetilde{\delta}_{1}$ such that $q \leq 10^{25}$ and

$$
q\left\|q \widetilde{\delta}_{2 i}\right\|>\frac{5 \cdot 10^{17}}{n \log n}
$$

then (12) cannot hold for $v, V \in \mathbb{Z}$.

Proof. Assume that there exist $v, V \in \mathbb{Z}$ which satisfy (12) and $\left|\Lambda_{i}\right|<$ $10^{-50}$. We recall that (12) implies $V>|v-1| n(\log n-\log 2)$. Let us fix $i$.

Let $p / q$ be a convergent of $\widetilde{\delta}_{1}$ with the properties given in the lemma. Multiplying (12) by $q$ and inserting $\delta_{1}$ and $\delta_{2 i}$ we get

$$
q\left|\Lambda_{i}\right|=\left|q \widetilde{\delta}_{2}+q\left(\delta_{2 i}-\widetilde{\delta}_{2 i}\right)+v\left(q \widetilde{\delta}_{1}-p\right)+v q\left(\delta_{1}-\widetilde{\delta}_{1}\right)+v p+V q\right|<10^{-25}
$$

Thus

$$
\left\|q \widetilde{\delta}_{2 i}\right\|<2 \cdot 10^{-25}+|v|\left|q \widetilde{\delta}_{1}-p\right|+|v| 10^{-25}
$$

and

$$
q\left\|q \widetilde{\delta}_{2 i}\right\|<2+|v| q\left|q \widetilde{\delta}_{1}-p\right|+|v|<\frac{5 V}{n \log n}<\frac{5 \cdot 10^{17}}{n \log n},
$$

which is a contradiction. The lemma is proved.

As $|v-1| \neq 0$ we have $V>n \log (n / 2)$, which implies $\left|\Lambda_{i}\right|<10^{-50}$ for $n \geq 25$ immediately. The same is true for $n \geq 8$ and $|v|>10$. In these cases we computed $\delta_{1}$ and $\delta_{2 i}$ with 50 decimal digit precision and found for all $n$, where $8 \leq n \leq 1700$, a convergent $p / q$ of $\widetilde{\delta}_{1}$ with the properties given in the lemma. For those values (12) cannot hold. We tested (11) for $8 \leq n \leq 25$ and $|v| \leq 10$ separately.

In the remaining cases $0<n \leq 7$ we used the method from Pethö and Schulenberg [PSch], but did not found any more solutions. Thus, Theorem 3 is proved.

For the computation we used PARI-GP on a notebook. The total computation time was about an hour.

\section{References}

[BD] A. Baker and H. Davenport, The equations $3 x^{2}-2=y^{2}$ and $8 x^{2}-7=z^{2}$, Quart. J. Math. Oxford 20 (1969), 129-137.

[BSt] A. Baker and C. L. Stewart, On effective approximations to cubic irrationals, in: New Advances in Transcendence Theory, Proc. Sympos., Durham 1986, A. Baker (ed.), Cambridge Univ. Press, 1988, 1-24. 
[BW] A. Baker and G. Wüstholz, Linear forms and group varieties, J. Reine Angew. Math. 442 (1993), 19-62.

[GyP] K. Györy and Z. Z. Papp, Norm form equations and explicit lower bounds for linear forms with algebraic coefficients, in: Studies in Pure Mathematics (to the memory of Paul Turán), P. Erdős (ed.), Akadémiai Kiadó and Birkhäuser, Budapest, 1983, 245-257.

[LMN] M. Laurent, M. Mignotte et Y. Nesterenko, Formes linéaires en deux logarithmes et déterminants d'interpolation, J. Number Theory 55 (1995), 285-321.

[LP] F. Lemmermeyer and A. Pethő, Simplest number fields, Manuscripta Math. 88 (1995), 53-58.

[LeP] G. Lettl and A. Pethő, Complete solution of a family of quartic Thue equations, Abh. Math. Sem. Univ. Hamburg 65 (1995), 365-383.

[M] M. Mignotte, Verification of a conjecture of E. Thomas, J. Number Theory 44 (1993), 172-177.

[MPR] M. Mignotte, A. Pethő and R. Roth, Complete solutions of quartic Thue and index form equations, Math. Comp. 65 (1996), 341-354.

[MT] M. Mignotte and N. Tzanakis, On a family of cubics, J. Number Theory 39 (1991), 41-49.

[P1] A. Pethő, On the representation of 1 by binary cubic forms with positive discriminant, in: Number Theory, Ulm 1987, H. P. Schlickewei and E. Wirsing (eds.), Lecture Notes in Math. 1380, Springer, 1989, 185-196.

[P2] -, Complete solutions to a family of quartic diophantine equations, Math. Comp. 57 (1991), 777-798.

[PSch] A. Pethö und R. Schulenberg, Effektives Lösen von Thue Gleichungen, Publ. Math. Debrecen 34 (1987), 189-196.

[T1] E. Thomas, Complete solutions to a family of cubic diophantine equations, J. Number Theory 34 (1990), 235-250.

[T2] - Solutions to certain families of Thue equations, ibid. 43 (1993), 319-369.

Département de Mathématique

et Informatique

Université Louis Pasteur

7, rue René Descartes

67084 Strasbourg Cedex, France

E-mail: mignotte@math.u-strasbg.fr

Erwin-Rohde-Str. 19

D-69120 Heidelberg, Germany

E-mail: hb3@ix.urz.uni-heidelberg.de
Laboratory of Informatics University of Medicine Nagyerdei Krt. 98 H-4032 Debrecen, Hungary E-mail: pethoe@peugeot.dote.hu 